\title{
User Haptic Experience and the Design of Drawing Interfaces
}

\begin{abstract}
Haptic feedback has the potential to enhance users' sense of being engaged and creative in their artwork. Current work on providing haptic feedback in computer-based drawing applications has focused mainly on the realism of the haptic sensation rather than the users' experience of that sensation in the context of their creative work. We present a study that focuses on user experience of three haptic drawing interfaces. These interfaces were based on two different haptic metaphors, one of which mimicked familiar drawing tools (such as pen, pencil or crayon on smooth or rough paper) and the other of which drew on abstract descriptors of haptic experience (roughness, stickiness, scratchiness and smoothness). It was found that users valued having control over the haptic sensation; that each metaphor was preferred by approximately half of the participants; and that the real-world metaphor interface was considered more helpful than the abstract one, whereas the abstract interface was considered to better support creativity. This suggests that future interfaces for artistic work should have user-modifiable interaction styles for controlling the haptic sensation.
\end{abstract}

\section{Author Keywords}

Haptic feedback, user experience, user perception, metaphors, evaluation.

\section{Introduction}

Matisse, when working on his paper cutouts was reported by Guichard Meili (1984) to have said: "Just feel it! ... It took a lot of research and many experiments to find it. I can become totally absorbed in working on this paper with scissors ..." This illustrates the importance of haptic sensation in creative activities, and its potentially positive effect on the user. It also suggests that an understanding of users' subjective experience could guide the design of haptic feedback in an artwork system.

Haptic interfaces in art-related applications have been reported as being able to improve users' performances and enhance their creative processes (Baxter et al., 2001; Kim et al, 2003; Shillito et al., 2001; Yeh, et al., 2002). However, work to date has focused on the realism of the haptic sensation, rather than on understanding the role of haptic feedback in creating a positive user experience for art-related applications, or on how users might choose between alternative haptic sensations in interactions.

In this paper we focus on user haptic experience in drawing. We examine current work that involves haptic sensation in drawing-related applications and identify ways to represent those sensations in interface designs, with a view to supporting the creativity of users. We present a study whose findings revolve around users' preferences and experiences of haptic feedback, considering in particular the role of haptic metaphors in supporting the interaction between users and drawing system.

\section{Background}

Haptic feedback is an aspect of the design of human-computer interactions that has potential to support user experience goals (MacLean, 2000). The haptic sensation perceived could influence users' preferences, and hence their acceptance of a new system. This has prompted us to explore how haptic feedback has been, and should be, presented in order to satisfy user experience requirements (Preece et al., 2002). 


\subsection{Haptic Sensation in Design Interface}

The most common approach that involves haptic feedback in art-related interfaces concentrates on realism of the haptic sensation (Baxter et al., 2001; Baxter et al., 2004; Kim et al, 2003; Yeh et al., 2002). For example, the painting applications dAb (Baxter et al., 2001) and IMPaSTo (Baxter et al., 2004) have been implemented to mimic real world objects and behaviours in terms of both the appearance of the paintbrushes on the interface and the haptic sensation felt. The visual cues from the virtual brushes on the interface are believed to help users to develop their own mental models for understanding how the system works. Such designs are exploiting knowledge transfer based on users' previous experience of similar interactions. Most of the existing systems use an abstract representation of real world objects at the interface for which the underlying haptic information mimics the real world sensation.

The other popular approach relating to haptics in art-related interfaces involves providing haptic feedback that does not resemble any specific real world tactile experience to the users (Guerraz et al., 2003; Lin et al., 2002; Snibbe et al., 1998). Such haptic sensations correspond to Masden's proposal (Masden, 2000) that system designers should try to exploit the power of the technology and provide opportunities not available in the real world. Dynasculpt (Snibbe et al., 1998), Texture Touch (Guerraz et al., 2003) and ArtNova (Foskey et al., 2002); Lin et al., (2002) are examples of systems that support creative artwork and that take innovative approaches to presenting underlying haptic information. In most cases (Guerraz et al., 2003; Lin et al., 2002), textual descriptors are provided on the interface to manipulate the haptic parameters of the system. Various buttons and slider bars are used for manipulating the visual image of a virtual object and its haptic properties, and the user can change some physical parameters of the haptic device in use.

Both approaches share a focus on technical aspects of haptic rendering rather than on how that feedback is experienced by the users. Also, neither line of work includes a discussion of how the appearance of the interface that represents the haptic feedback is selected. The work reported in this paper has taken a more systematic approach to presenting haptic information on an interface under user control. The focus of this work is on how haptic design supports user drawing activities and influences the user experience.

\subsection{Motivation for the Research}

Because there has been relatively little research on user experience of haptic interactions, designers have little information on users' perceptions of haptic feedback in a design. Users may not like or accept a haptic drawing application if the features do not support their requirements and do not offer advantages compared to real world drawing interaction (Scali et al., 2002). User experience when interacting with a haptic drawing interface needs to be better understood. Baxter et al.'s works (e.g. Baxter et al., 2001; Baxter et al., 2004) that provide different haptic sensation from various types of paintbrushes indicate that such additional complexity of haptic feedback stimulates artists' creative minds; thus, motivates our research. Representing haptic information in an interface is particularly challenging because of users' individual preferences. The research questions motivating this work included whether having user-controllable haptic feedback improve the user experience, and, if so, what a suitable haptic metaphor might be. Our focus is on user experience in order to understand features of haptic feedback in a drawing application.

In the following sections, we outline the initial study, which identified a vocabulary of haptic cues, then the design of our haptic drawing prototypes, an overview of the implementation, and the results of the evaluation study that is the main focus of this paper. We conclude with a discussion of the implications of this research for future work on user experience of haptic interfaces and the design of systems to support drawing.

\section{Haptic cues for supporting interaction design in the drawing domain}

There have been many attempts to include haptic cues such as softness, hardness, and stiffness in interface designs; however, it is not clear how these cues are chosen and when they should be applied for interactions. As noted in Yu and Brewster (2003), the haptic modality is very context dependent: the choice of haptic feedback depends on the particular application domain. Therefore, in order to develop a system that reflects a user-centred understanding of haptic experiences when drawing, a qualitative study with artists was conducted. This study set out to establish a set of tactile features that are significant in the drawing domain. This work is reported fully in [reference removed for blind review purpose], here we present a focused summary of the method and findings. 


\subsection{Method}

21 traditional artists took part in the study (13 females and 8 males). 2 of the artists paint for a living, while 15 were undergraduate and 4 were postgraduate students from the Slade School of Art at University College London. All the artists were interviewed and the conversations were audio recorded. During the interviews, the artists were given a collection of 9 pen-like tools ( 4 pens, 3 pencils, a crayon and charcoal, selected to represent a broad range of tactile experiences) to work with. They were asked to describe the tactile sensation and the visual appearance of each tool whilst holding it in their hands. Using the tool, they were asked to do free drawing or writing on two different types of textured paper. During their interactions, they were asked to talk aloud about the tactile sensation they experienced and the appearance of the mark produced when they drew with each tool on the paper. They were also encouraged to talk about any similar experiences using drawing tools in their daily work.

The recorded conversations were transcribed, and the vocabulary that was used by the artists was identified. These vocabularies were examined to identify the terms used by each artist across all the 9 pen-like tools and two paper types. From the vocabularies, any similar terms were grouped into the same category. The groups obtained were further classified based on the actions made by the artists, so as to determine at which stage of interaction a particular haptic cue should be applied in the interface design. To ensure consistency of the terms used by the artists when describing each tool, an orthogonal data analysis was performed. In this, the transcribed data was transformed into 9 different sets based on the tools used in the study. The terms used by all the 21 artists to describe the tactile sensations when using each tool were identified. These terms were cross-examined to assess their internal consistency. No major inconsistencies were found.

\subsection{Study Results}

Three types of interaction were identified: that between the hand and the tool (as experienced when holding the tool); that between the tool and the surface (experienced when pressing or pushing the tool over the surface); and that between the hand and the surface (experienced when smudging a line on the surface). Since the haptic system used in the evaluation study (to be described later in this paper) was a PHANTOM desktop device with a pen-like interaction tool (http://www.sensable.com/haptic-phantom-desktop.htm), we focused on the user experience of interaction between the tool and the surface.

The 'tool and surface' interaction involves two main actions made by the artists: "press" and "push". In this analysis the action "press" is considered as a passive touch (Klatzky and Lederman, 2002). This action provided information about the hardness and softness of an interaction as the tool touches the surface of the paper. In contrast, the action "push" involved active haptic exploration (Brewster, 2005). The artists applied force and made a mark on the paper. The "push" action provided information about the surface texture of the paper. It also produced friction between the tool and the paper, resulting in the following descriptions: Soft; Hard; Sticky; Dry; Waxy; Powdery; Smooth; Glide; Flows; Silky; Velvety; Creamy; Stiff; Sharp; Scratchy; Bumpy; Rough.

These tactile sensations were grouped into three dimensions: bumpiness, scratchiness and stickiness. These labels were derived from the root names of the tactile sensations described by the artists. From the user data, smoothness was identified as a neutral point of these three dimensions (i.e. that which occurred when bumpiness, scratchiness and stickiness were all low).

The cues and their neutral point were validated using a closed card sorting technique, involving 15 participants. Participants were given cue cards, each with a tactile cue obtained from the study written on it. The evaluators were asked to place the cards according to the appropriate group headings. A cue card could be placed into more than one group or may not necessarily belong to any of these three groups. In such cases, the evaluators were asked to formulate their own group title. All evaluators were asked to comment on the appropriateness of the labels given to the three groups. Almost all evaluators agreed with the naming of the three haptic dimension cues to represent the vocabularies of tactile sensation for a drawing interactions provided to them. Only one evaluator had a reservation about the term "scratchiness": she argued that the term "roughness" would be more appropriate.

\section{Representing haptic information}

As outlined in Section 2.2, one of the core questions motivating this research was what mediating representation would be appropriate for the user to control the haptic feedback. Haptic feedback involves abstract information that can be a challenge to interaction design. Reification by exploiting a suitable metaphor (Blackwell, 1998; Blackwell, 2006) could assist in making this abstract haptic information concrete. Besides making the information "tangible", options also need to be provided to meet users' individual haptic preferences (Yu and Brewster, 2003; Yu et al., 2001). 
As discussed above, haptic feedback in a computer drawing environment could either be presented as being similar to that in the real world ("isomorphic") or different from reality. This exploits the two fundamentally different roles of metaphor namely to express something by building on the similarity between the two referents or to convey something new by emphasising the dissimilarities (Masden, 2000).

A concrete real world object-based metaphor was considered a promising candidate for a design option because of its familiarity and attractiveness to most types of users, especially for first time and casual users of a system (Vaananen and Schmidt, 1994; Wells and Fuerst, 2000). In the case of a drawing application, the tools commonly used such as pen-like tools and different kinds of paper could be among the possible choices to be considered as interface metaphors. Such selections could be made from those used in the first empirical study. Familiarity with the objects may help the user anticipate the kind of haptic feedback to expect from the drawing tools. This interface design option satisfies the first role of metaphor (as emphasising similarity).

To ensure user familiarity with the metaphor used, it was decided to base the alternative design on the empirically derived vocabulary describing haptic sensation. The dimension cues "scratchiness", "bumpiness", and "stickiness" were selected as the textual information that appears on the interface and is implemented in the underlying haptic functionality. The fact that this does not represent any real-world objects means that the haptic information for the computer environment may not correspond to the haptic sensation experienced when using any drawing implements in the real world. This satisfies the second role of metaphor (as emphasising contrasts).

\section{Implementation of haptic drawing interfaces}

Three different interfaces were developed, all based on the same core haptic system. The main idea of the system is to enable users to feel the haptic cues during their interactions (i.e. when drawing activities take place). The haptic device used was the PHANTOM desktop. The graphics objects and haptic codes were created using the OpenGL API, and GHOST API, respectively. The simulation uses the contact force model as used in GHOST SDK.

\subsection{Graphical Interface}

There are three main components of graphical interface in the system, namely: the drawing paper, PHANTOM cursor, and the lines (images) drawn.

\subsubsection{Drawing paper}

The implementation of the drawing surface in the prototype involves creating a (visual) drawing paper and a (haptic) paper base (see Figure 1).

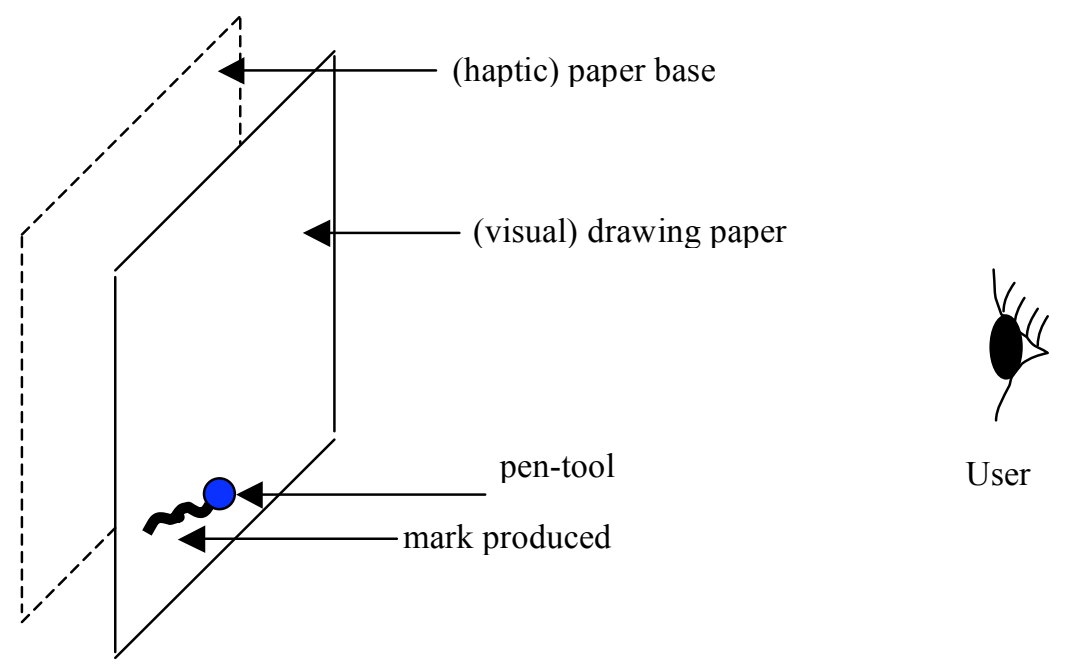

Figure 1: (Visual) Drawing paper \& (Haptic) Paper Base 
The (visual) drawing paper is an area on the interface where the mark will appear during the drawing interactions. This area will be texture mapped with visual images of a drawing paper i.e. smooth or textured, depending on the choice made from the menu bar. The (haptic) paper base is designed to create a feeling that drawing is done on a hard surface. This will stop the users from going 'through' the paper. For this reason the (haptic) paper base is placed 'behind' the (visual) drawing paper i.e. out from the user's viewpoint and is not being graphically rendered. There is a gap created between the (visual) drawing paper and the (haptic) paper base to allow the implementation of the force feedback field in the design. This is discussed in Section 5.2.1.

The (visual) drawing paper and (haptic) paper base are created using OpenGL and GHOST APIs, respectively. A square of size $120 \mathrm{~mm} \times 120 \mathrm{~mm}$ is created to represent the (visual) drawing paper. The centre of the paper is positioned at coordinates $(0,0,-65)$. A cube of size $140 \mathrm{~mm} \times 140 \mathrm{~mm} \times 140 \mathrm{~mm}$ is created and translated so that one of the cube faces is positioned parallel and behind the square (i.e. hidden from the user's view). This cube represents the (haptic) paper base.

A simple illustration of the (visual) drawing paper and the (haptic) paper base from the top view (y-axis) is presented in Figure 2.

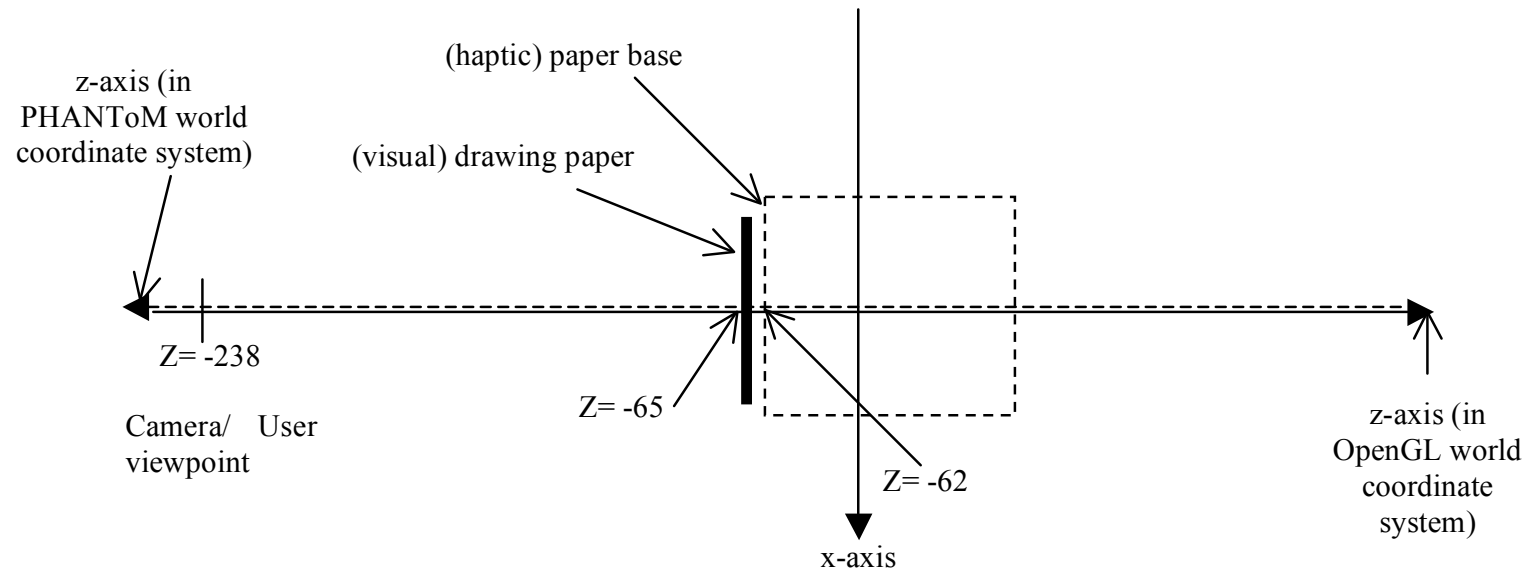

Figure 2: (Visual) Drawing paper \& (Haptic) Paper base - Top View

\subsubsection{PHANToM cursor}

A triangle was created to function as the PHANTOM cursor. The PHANTOM position ( $\mathrm{x}, \mathrm{y}$ and $\mathrm{z}$ value) is attached to this triangle so that the coordinates for both PHANTOM and triangle can correspond to one another. As this research is focusing on haptics rather than visualisation, the same triangle is used as a visual representation of the pen-like tool tip throughout the prototype. This triangle emulates as closely as possible the pen-like tool tip behaviour i.e. the tactile sensation felt (when drawing) of each pen-like tool chosen on the interface. The default pen-like tool for this prototype is a thin-tip pencil, which feels hard when used for drawing.

\subsubsection{The lines (images) drawn}

The lines or images drawn on the interface are a result of an interaction made during a drawing activity. These images appear when the PHANTOM cursor touches the surface coordinates of the drawing paper. The thickness of these lines corresponds to the pen-like tool chosen. For example, a thin line is the visual result following from an interaction using a $3 \mathrm{H}$ pencil on smooth paper.

The fact that this research focuses on haptics makes the visualisation secondary to the investigation. Consequently, when implementing the system, while the haptic feedback attempts to mimic as closely as possible the cues presented in Section 3.2., only a simplification of line thickness is made with respect to the pen-like tools used. For the implementation purpose in this research, a pencil with a thin tip will make a small, light line while a wide tip will produce a slightly bigger and darker mark. Regardless of the tip size, a pen will produce a bigger mark than a thin tip pencil. Both crayon and charcoal will make the biggest line as compared to a pencil and a pen. In the actual implementation three different sizes of line are used. ' $3 \mathrm{H}$ pencil', '2B pencil', and 'ball-point pen' have the same thin line; 'graphite pencil', 'felt-tip pen', and 'roller-ball pen' have a 
medium size line; 'crayon', and 'charcoal' have the same large line. These sizes apply to both on the 'smooth' and 'rough paper'.

\subsection{The Haptic Effect}

This section discusses the rationale of the haptic implementation and how the dimension cues highlighted in Section 3.2 are integrated into the prototype.

\subsubsection{The Rationale}

The haptic system provides users with a tactile experience during their drawing interaction on the interface. It involves $2 \mathrm{D}$ drawing in a 3D environment. The system follows the analogy of drawing in the real world (see Figure 3(a)) in that users can draw on the surface paper and feel the tactile sensation of the interaction. This analogy is motivated by Nakakoji and Yamamoto (2003) who considered drawing as a representation of an object. They state that the act of holding a drawing tool, i.e. pen or pencil, and moving this tool while pushing its lead to obtain a mark on a piece of paper is an interaction. In such a case a drawing interaction can be equated to an object that should contain the haptic information in question. With this idea, the sensation of different haptic force feedback when using various pen tools on paper types could only be felt during a drawing interaction. The users do not feel a different force if they draw over a previously drawn line. Figure 3(b) shows an example of a line drawn on the drawing surface and the surface texture profile assigned to the haptic paper base.

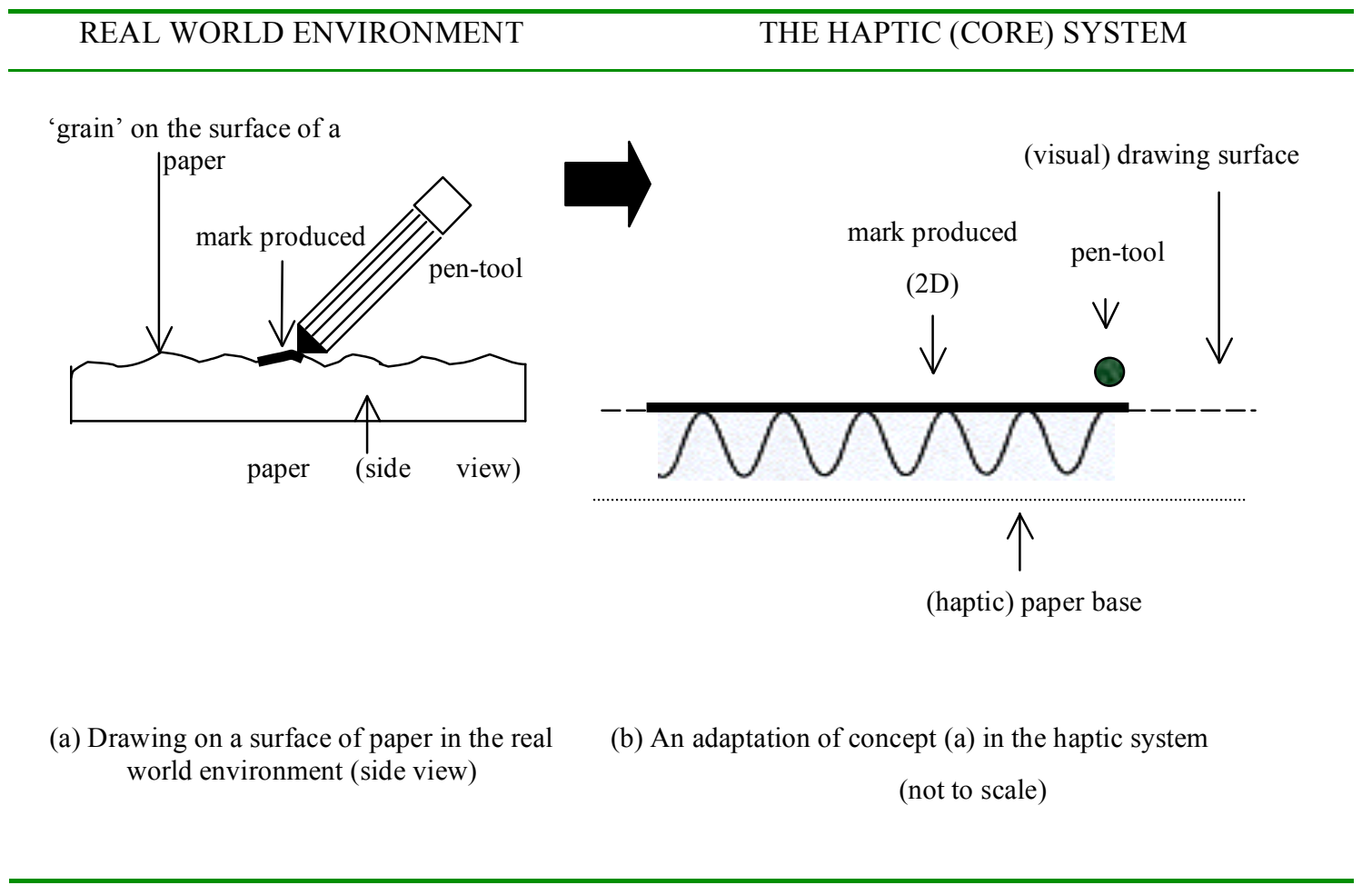

Figure 3: An analogy of a drawing interaction

In Figure 3(b) the surface texture profile is represented by a series of sine waves that is implemented in the gap between the visual drawing surface and the haptic paper base. The sine waves could easily allow changes on the surface texture to be made in order to simulate various haptic sensations. In this system, the implementation of haptic sensation is inspired from the idea that interface designers use findings on how people perceive and manipulate active and passive exploration of touch (Klatzky and Lederman, 2002) to simulate haptic behaviour for interactions. By manipulating the surface texture profile of the drawing surface, an illusion of interacting on various media with drawing implements could be created. In other words, by changing the surface texture profile upon selecting a pen-like tool and paper type combination we are able to simulate various tactile sensations when, say, interacting using a pencil on smooth paper and using crayon on rough paper. Burdea (2000) supports this idea whereby he highlights that surface texture can be used for physical modelling on the smoothness and roughness of a virtual object. McGee et al. (2001) proved that texture roughness could be discriminated by changing the 
surface texture profiles: haptic perception is a result of an active exploration whereby users understand and interpret the experience they have just felt.

\subsubsection{Implementing the Haptic Effect}

In general, the simulation of haptic feedback in this prototype depends on the parameter settings provided by GHOST SDK. Consequently, the prototype follows the contact force model as used in GHOST SDK. A user can feel this feedback as they explore the PHANToM cursor on the surface texture of the (haptic) paper base. From Section 3.2., three haptic dimension cues, i.e. bumpiness, scratchiness and stickiness and a neutral point, smoothness, have been considered to be important for the integration.

The integration of the three haptic dimension cues and their neutral point in the system involved an adaptation of Wall and Harwin's (2000) 2D sinusoidal wave model implemented on the paper surface. In this prototype the sinusoid is implemented on the $\mathrm{X}$ and $\mathrm{Y}$-axes of the haptic paper base. The variation of haptic sensation implemented for the drawing surface is done by manipulating the frequencies and amplitudes of the sine waves, and manipulating the parameters supported by PHANToM, which are the coefficients of static and dynamic friction, and values for stiffness and damping factors. According to Castle et al. (2002), the stiffness and damping parameters act as internal friction of an object.

In integrating the haptic effects, all parameters are treated inter-dependently. It should be emphasised that the exercise described in this section only provides a simple guideline for such implementation. The basic concept to implement the haptic effects is as follows:

\section{Bumpiness (rough; rough/ bumpy)}

By manipulating the amplitude and frequency of the waves a bumpy feeling could be felt. This could be achieved for example by having a high amplitude and low frequency of the waves. This approach is justified by McGee's (2001) research work on surface texture roughness.

\section{Stickiness (sticky, creamy, velvety)}

The stickiness sensation is implemented by manipulating the friction parameter settings. This idea is replicated from approaches used by Foskey et al. (2002), Castle et al., (2002), Iinuma et al., (1999) and Yu et al. (2001) who manipulated the friction values to get a stickiness effect. Keeping the coefficients of static and dynamic friction high creates a 'sticky' feeling of the interaction.

\section{Scratchiness (hard, dry, stiff, sharp/scratchy)}

Manipulating the frequency of the sine waves implemented on the haptic paper base simulates the scratchiness effect. This is related to the bumpiness effect as well. Keeping the amplitude low, making the frequency high, and varying the frequencies of the $\mathrm{X}$ and $\mathrm{Y}$-axes of the haptic paper base creates a scratchy sensation for the drawing interaction. In this system, the scratchiness effect has been implemented through trial and error. This effect was tested in a formative evaluation performed throughout the development process.

\section{Smoothness (smooth, silk, flow, glide)}

The smooth sensation is implemented by manipulating the friction parameter settings. Foskey et al. (2002) stated that the lack of overly friction rendering can create an overly smooth feeling during a haptic interaction. Yu et al. (2001) used this approach when implementing a slippery sensation on a simple haptic graph. By keeping the coefficients of static and dynamic friction low could create a smooth sensation. This is in contrast with implementing the stickiness effect.

It should be noted that the initial implementation of the system is taking the form of an object-based metaphor interface design in which the pen tools and paper types combinations are involved. In such a case, the three dimension cues need to be manipulated for each of the combinations. The datasets involved in the object-based metaphor interface design could be used as a basis to implement the haptic effect for a textual description metaphor interface design option. 


\subsection{The Three Interfaces}

In order to establish whether having user-controllable haptic feedback was perceived by users as being beneficial, two haptic interfaces were developed based on the "real world" metaphor: one with fixed haptic feedback ("Interface A") and the other with realistic haptic feedback ("Interface B"). In the case of the fixed haptic feedback implemented (for "Interface A"), users could only feel the smoothness sensation throughout the interaction using all the 16 tool-paper combinations (derived from the 2 paper types and 8 pen tools shown in Figure 5). Such a feedback is chosen to reflect the neutral point of the three dimensions of tactile sensation (i.e. bumpiness, scratchiness and stickiness) as described in Section 3.2. The visual feedback (e.g. the line width applied to each tool) was the same in both interfaces. The screen layout and tool palette for Interfaces A and B are shown in Figures 4 and 5. Since the focus of this work was on user experiences, rather than developing optimum haptic simulations of 16 tool-paper combinations, the approach taken for Interface B was to design the haptic feedback iteratively, to be a reasonable approximation to the natural feel of each drawing tool on each paper type, rather than investing all the research effort in ensuring near-perfect haptic simulations. Some of the consequences of this approach are discussed in Section 6 when presenting the evaluation study findings.

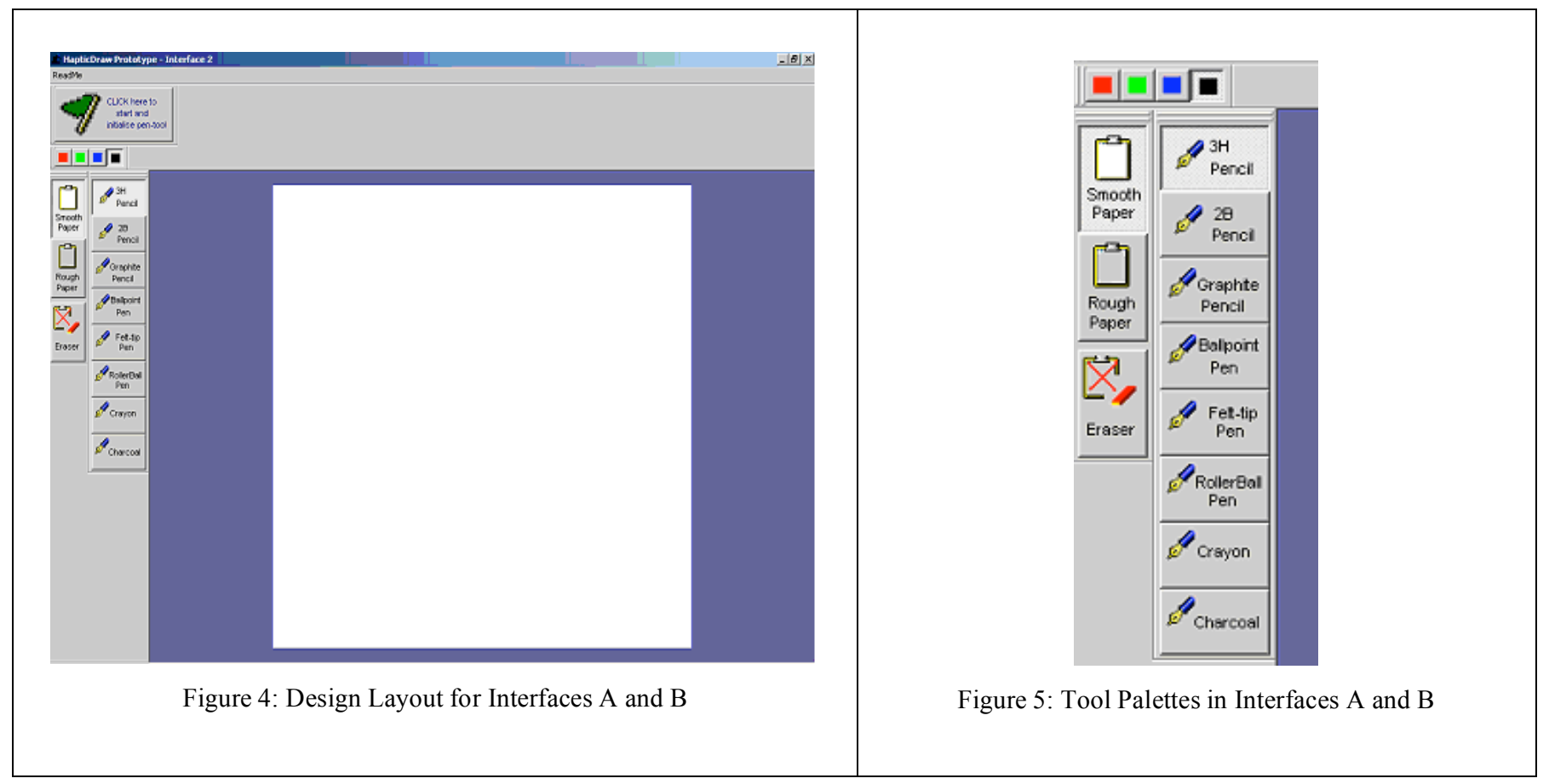

In order to evaluate the relative merits of the "real world" metaphor as compared with a "dimension cues" metaphor, a third interface ("Interface C") was also developed. The interface design is shown in Figure 6. The user is able to change the parameter levels of "scratchiness", "bumpiness", and "stickiness" (see Figure 7) to get different haptic effects. The datasets used for Interface B formed the basis for the haptic feedback in this interface but the different possible combinations do not correspond directly to any real world combinations of tool and surface. In this case, none of the settings for datasets in Interface B is identical to those in Interface C. Buttons were chosen rather than sliders to make the interaction styles of the interfaces as similar as possible for the comparative evaluation. 


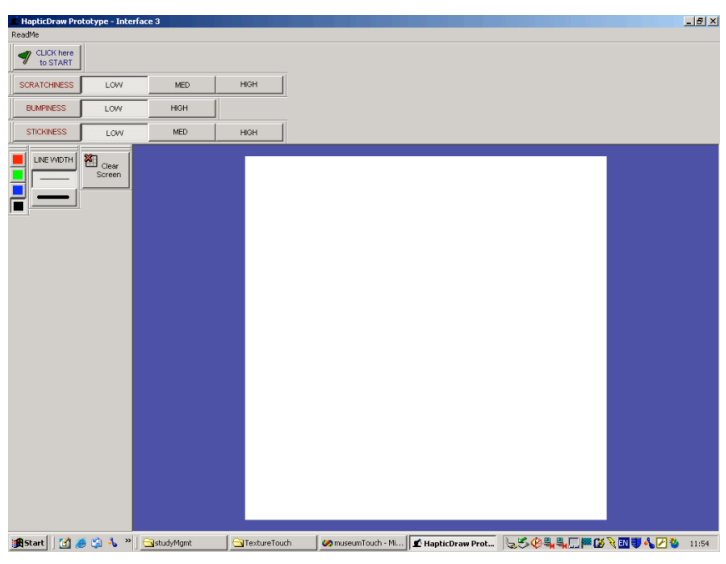

Figure 6: Design Layout for Interface C

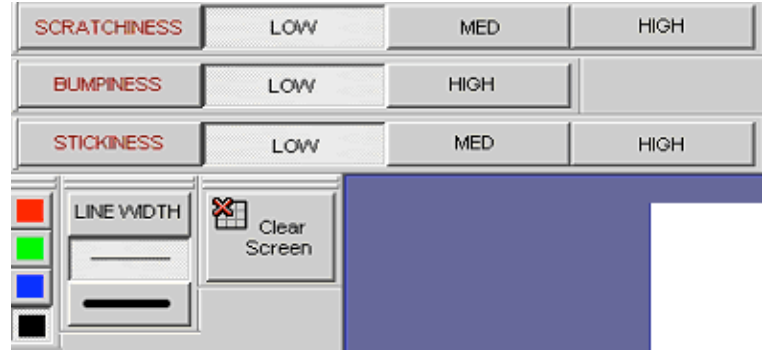

Figure 7: Tool Palette in Interface C

\section{Evaluation Study}

As discussed above, the central questions being addressed in this research are whether users perceive haptic feedback as contributing positively to their experience of drawing, and what the relative merits of the two alternative interface metaphors described above are. Subsidiary questions included how having a real-world metaphor influenced user expectations and which tactile sensations were preferred, and why.

\subsection{Method}

24 traditional artists took part in the study (14 females and 10 males). 7 of the artists paint either for pleasure or for living, while the rest were students from the Slade School of Art at University College London (4 undergraduate and 13 postgraduate). All artists were paid for their participation. None of them had participated in any earlier studies. They were provided with training on using the PHANTOM before the study.

In the study, a counterbalanced repeated measures design was used. Each artist was given about 10 to 15 minutes to interact with the first interface. They were asked to do free drawing using the PHANTOM, and describe out loud the tactile sensation felt. They had to try all combinations on each interface (the researcher, using a mouse, did the combination changing at the user's request). Next, a debriefing session was conducted. The artists were asked which combination(s) they liked to interact with most and to give reasons for this choice. For example, the artists were asked for the pen-tool metaphor interface (Interface B): In terms of the tactile sensation, which of the pen-tool and paper type combinations do you prefer to use? And why? They were allowed to request any combinations they wished to try again to confirm their judgments of the haptic sensation in this preference exercise.

Next, the artists were given the second interface to evaluate. All procedures were replicated. Towards the end of the evaluation they were asked which of the two interfaces they preferred to interact with in terms of the tactile sensation and why. At this stage an interim preference was obtained.

Finally, the artists were given the third interface to evaluate; again, they were asked to try all combinations of cues available, and the same steps were followed. After the evaluation, the artists were asked for their preference between the previous interface that they preferred and the third interface, and their reasons for this. This preference was used as the inferred final preference in the study. In retrospect, this approach to questioning meant that in a small number of cases (where the interim and final preferences were the same), the relative preferences of the other two interfaces were not established, so that the final analysis is sometimes based on fewer than 24 data points (see below). This approach is taken since it is not our main intention to find out the best out of the three interfaces evaluated. Rather, this evaluation is more focusing on eliciting users' subjective experiences of the three interfaces in terms of the haptic feedback provided. 
An overall debrief was conducted to rank the three interfaces based on the artist's preference in terms of the haptic feedback. User experience criteria based on Preece et al. (2002) were used for this debrief.

The interviews were transcribed verbatim from the audio recordings, yielding twenty-four data sets. The study resulted in both quantitative and qualitative data. The quantitative data consists of the artists' preference towards the interface, and ranking of interfaces with respect to user experience goal criteria and tactile cues during the interaction, while the qualitative includes the articulated reasons for such preferences, vocabularies used to describe the tactile sensation in Interface B, and any other remarks pertaining to the study that were voluntarily suggested by the artists during the evaluation. It should be noted that only a simple quantitative analysis such as counting the number of preferences was involved in this exercise. The main intention for this quantitative data is to provide a foundation for presenting the artists' subjective experience from the study. No statistical data on the ranking of the three interfaces was involved in the analysis. Qualitative findings whereby the reasons a particular haptic interface is preferred based on artists' perspectives provides a more appropriate assessment; this may limit generalisability of the study results.

\subsection{The perceived realism of haptic feedback (Interface B)}

One factor that was likely to affect participants' perceptions of the three interfaces was the perceived realism of the haptic feedback in Interface B.

Using a similar technique for identifying vocabularies of haptic cues to that used in the first study, the transcribed data from the 24 artists who took part in the evaluation study were examined. The resulting vocabularies were compared against those identified in the initial empirical study.

It was found that artists used a similar vocabulary to describe their tactile experience in the computer drawing interaction to that in the real world situation. Terms such as 'soft', 'waxy', velvety', 'stiff', 'scratchy' and 'rough' featured in both sets of interactions. There was no perceptible difference in terminology between those who used Interface B after C and those who used Interface B before C. The tactile sensation obtained from the same pen-tool and drawing paper combination was also consistently described or associated with a similar kind of expressions by most artists. In other words, there was agreement over what haptic features each pen-tool and paper type combination had. The study findings show that, at least to some extent, haptic experience when drawing and sketching in the real world is transferable to a computer environment.

However, there were some small but significant discrepancies in the language used. The terms 'powdery', 'silky', and 'creamy' were identified in the initial study, but did not feature in the second (PHANTOM) study. These terms suggest a rich tactile sensation that apparently was not achieved in the PHANTOM environment. In contrast, the terms 'flat' and 'harsh' featured in the vocabularies when using the PHANTOM but not in the initial study. These findings indicate that although the artists found the simulated haptic feedback felt similar to the real world there are still some haptic elements that they perceived to be mechanical and unnatural. The contrast was greater for some of the simulated tools than others, as discussed below.

\subsection{The perceived value of user-controllable haptic feedback}

The first question raised is whether (and why) users value having control over the haptic feedback. This question has been explored by comparing Interfaces A and B. Twenty of the participants expressed a preference between these two interfaces, as shown in Table 1.

\begin{tabular}{|l|l|}
\hline Prefers A & 3 \\
\hline Prefers B & 15 \\
\hline No preference & 2 \\
\hline
\end{tabular}

Table 1: Interface Preferences (Interfaces A \& B)

Table 1 indicates that the preference for Interface B rather than Interface A. The qualitative data was analysed to probe reasons for this preference.

One clear factor was that the real world object-based metaphor interface led to an expectation of how the tactile sensation should feel when interacting using the pen tool on paper type combinations provided. Most artists commented on the fact that there was no difference in terms of the tactile sensation perceived from one pen-tool to another, or between the smooth and rough papers, in Interface A. This is not what the artists had expected based on the metaphor suggested. For example, when interacting with Interface A, Artist 8 said: "If I click on charcoal I have this tool in my hand and this tool is I would imagine intended to simulate an artist holding a pen, holding this stick or paint. Then I would expect the charcoal to feel a bit like charcoal." 
The fact that the haptic sensation felt the same throughout the interaction with Interface A, regardless of the pen-tool and paper type combination chosen, created difficulties in assessing the haptic feedback to determine whether the sensation perceived felt similar to that in the real world. By just experiencing the tactile sensation, an artist could not guess the type of drawing tool they are using. For example, Artist 13 said: "Most of them are hard to define which one is which. If I wouldn't see these, er, buttons of $3 H$ pencil."

In other cases, with Interface A, there was a breakdown in the metaphor. The fixed haptic feedback in Interface A was perceived as similar to ball-point pen or $2 \mathrm{~B}$ pencil in the real world. For example, Artist 13 noted that the tactile sensation when assessing the ball-point pen on Interface A felt similar to the actual material in the real world: "Pretty much the same feeling as material like ball-point." However, he also perceived the roller-ball pen as having a similar tactile sensation to what he experienced when evaluating the ball-point pen: "It's like ball-point pen."

The variation of haptic sensation in Interface B resulted in an enjoyable haptic interaction. In the case of Artist 11 , he was fascinated by the fact that the physical pen-tool (i.e. PHANTOM) is able to mimic various haptic effects of pens for drawing. When asked whether he preferred to interact with Interface A or B, he replied: "Definitely Interface B! I think the whole thing is interesting. What provokes me to keep drawing is that if I can have different feelings. "He further commented on the ability to use the device to replicate many pen-tools: "If I stick to the same tool but I got a different feedback come out from the same identical pen that would be like a magic pen!"

The realism of Interface B was also valued. For example, when comparing Interfaces A and B, Artist 2 said that she preferred the latter. In this case, she commented on the tactile sensations of 'roller-ball' and 'felt-tip' pens: "I think this one is just much more realistic experience. The 'roller-ball' feels like roller-ball because it feels less sticky whereas the previous one (pause). Here, it is more different in using 'felt-tip' and 'roller-ball' but that one, there isn 't." Similarly, Artist 8 expressed disappointment at the absence of the expected tactile feedback in A: "Having gone through this process, it almost feels a bit disappointing not to feel the bite of charcoal."

There is evidence from some artists' responses that haptic feedback is able to engage their expressive feelings. According to Artist 1: "Vibration is very important to draw because you can think of many things with feeling, expressing feeling." This suggests that tactile feedback could be associated with the generation of ideas. Artist 11 said he could perform more efficiently: "For me it's like I can do quickly with different kinds of feeling." The importance of haptic feedback presented in Interface B is clear, particularly because this interface is preferred by many artists even though a less than perfect haptic simulation is used. Artist 11 elaborated: "On this pen, apart from I can't touch the screen I feel like, er, this pen is fulfilling, well, only $40 \%$ what I would like to achieve for the reality".

\subsection{User preferences of the alternative interface metaphors}

The second question raised is whether (and why) users prefer the real-world or abstract metaphor. This question has been explored by comparing Interfaces B and C. Twenty-two of the participants expressed a preference between these two interfaces, as shown in Table 2.

\begin{tabular}{|l|l|}
\hline Prefers B & 9 \\
\hline Prefers C & 10 \\
\hline No preference & 3 \\
\hline
\end{tabular}

Table 2: Interface Preferences (Interfaces B \& C)

In this case, there is no measurable difference in preference between the two interfaces.

As noted above, the real-world metaphor led participants to have particular expectations of the haptic feedback. In contrast, the artists did not expect Interface $\mathrm{C}$ to deliver a haptic sensation that corresponded to a real world object. This led to some of the artists to prefer Interface $\mathrm{C}$, as it did not generate expectations that might then be violated by a less-than-perfect implementation. For example, Artist 1 explained his preference for Interface $\mathrm{C}$ rather than B by saying: "If it has a particular name or pencil or ball-pen or something like that, I tend to compare it with the real one. But because it has the general topic maybe scratchiness, bumpiness or something, yes, it's very general kind of feeling. I don't have to compare with the actual thing." Similarly, Artist 12 preferred Interface C, saying: "I prefer just turning to what actually I can choose."

Conversely, other participants valued the familiarity of the real-world metaphor. For example, Artist 8 remarked: "I still like the idea of being called 'charcoal'. Similarly, Artist 15, commenting on Interface B, remarked: "It's virtual but I can feel that I'm drawing with 'crayon', which is quite interesting how my mind associates with the virtual drawing. ” It would seem that the haptic and visual metaphors support one another in Interface B and provide associations.

Another theme that emerged for Interface $\mathrm{C}$ was the value of being able to directly manipulate the relevant underlying parameters. For example, Artist 10, who did the task sequence of Interface A then C, said: “This one, I prefer. This one I can 
control right, how I want to do it." Similar comments were made by others, and this control led to a preference for certain haptic cue combinations. For example, Artist 19 who prefers Interface C rather than A, said: "Interface A wasn't that good; Interface C, I just like that 'medium-low-low' thing. I think this is better. That called scratchiness; because I can set the pressure I want to draw rather than imitate a kind of material I want." We return to the theme of which combinations of parameters are preferred by different users below.

The ability to directly manipulate parameters made the haptic interaction more interesting for some artists, as they could experiment with the haptic feedback. Artist 6, who preferred Interface $\mathrm{C}$ as compared to Interfaces A and B, said: "I do like the extremes like the stickiness. I can't think of the reality the way you would be able to mimic those resistance so therefore I probably want more variety of it to try to make, to experience drawing in a different way. This is quite an interesting thing." To him, mimicking reality may not always be necessary: "media not necessary thing to create an end point." This was also noted by Artist 23, who expressed an element of freedom with Interface C: "generally it allows artists to be freer and be more creative."

Even though none of the dataset for haptic feedback implemented in Interface $\mathrm{C}$ is similar to those in Interface B, the feedback felt when the artists tried the combinations of cues has been associated to the tactile sensation when using some specific pen tools in the real world environment. Several artists were almost certain that the combinations of low-hi-hi (i.e. low scratchiness, high bumpiness and high stickiness) feels like drawing using a crayon. For example, Artist 13 said "more

like a crayon", and Artist 21 remarked, "Feels like crayon or something." However, the haptic sensation for each of other combination cues has been associated to not necessarily to one particular pen tool. As an example, the combination of lowlow-low (i.e. low scratchiness, low bumpiness and low stickiness) was perceived by Artists 12 and 23 as interacting using a pencil but the haptic feedback felt like using a roller-ball pen by Artist 21. Similarly, the tactile sensation for combination cues hi-hi-low (i.e. high scratchiness, high bumpiness and low stickiness) was perceived as a charcoal (Artists 5, 9, 22), a ball-pen without the ink (Artist 13), and chalk (Artist 21). With the exception of low-hi-hi combination, the findings indicate no definite association between the combination cues and the tactile sensation from pen tools in the real world. The artists tend to rely on their previous individual haptic experience in the real world when making such an association.

Despite artists' preference in the ability to experiment the combination cues, some artists had difficulty relating to the abstract parameters in Interface C. For example, Artist 18 commented on Interface C: "I cannot understand what 'scratchiness' is and I cannot understand what 'bumpiness' is; unless I try. ' Similarly, Artist 2 preferred Interface B because she found the metaphor used in Interface C difficult to comprehend as she tried to relate the haptic experience to the real world drawing interaction. "I prefer the paper one because it feels the paper more. This to me is what does this mean? Material and pencil tools?”.

\subsection{User Experience Criteria Preferences of Interfaces}

To better understand the qualities of the three interfaces, the artists were asked to rank them with respect to established user experience criteria (Preece et al., 2002). The number of times an interface or a combination of interfaces was rated as the preferred with regard to a particular criterion was counted (equal rankings were counted as 0.5 each for two, or 0.33 if all three interfaces were ranked the same). A graphical representation of this information for all ten criteria is presented in Figure 8. These results should be considered indicative rather than statistically significant. 


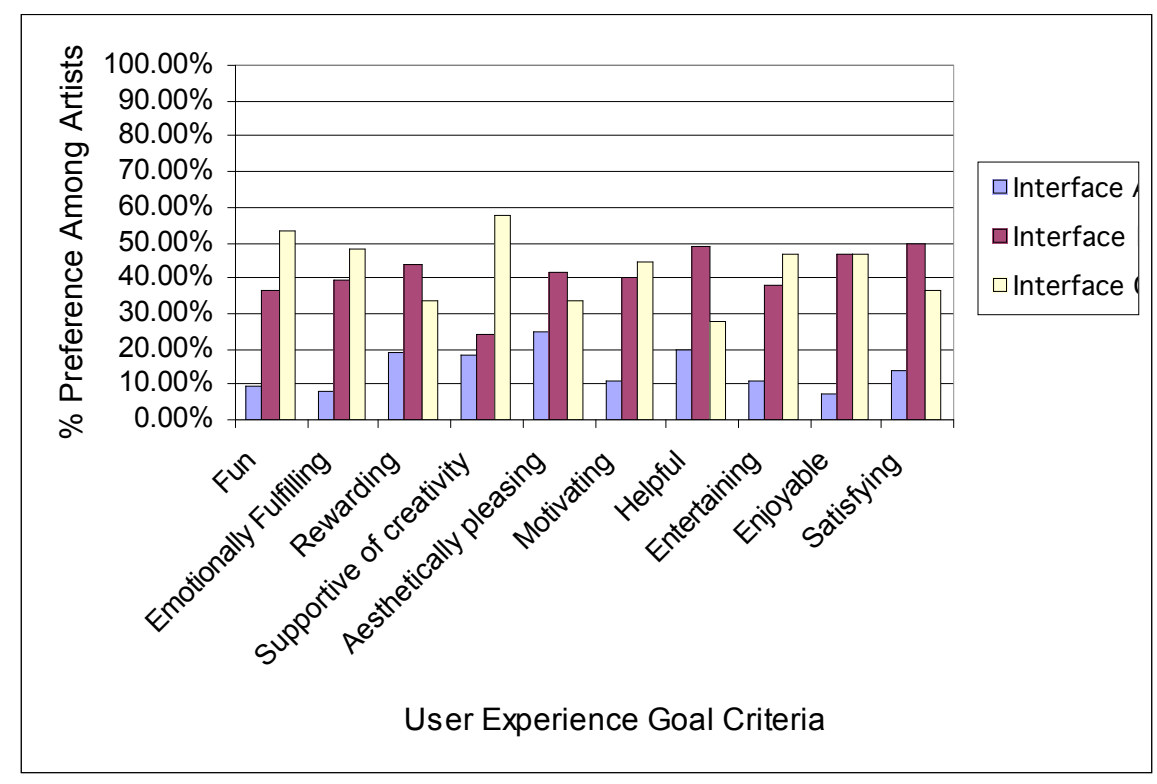

Figure 8: Ranking of Interfaces

Interfaces B and C were consistently rated higher than Interface A for all criteria. This indicates that Interface A, which has the same haptic feedback throughout an interaction, was not favoured when assessed against the user experience goal criteria. This is consistent with the result that the majority of artists preferred the interfaces with user-controllable haptic feedback.

As shown in Figure 8, Interface $\mathrm{C}$ scored highest on the 'supportive of creativity', 'fun', 'emotionally fulfilling', 'entertaining', and 'motivating' criteria. Conversely, Interface B was rated more highly for being 'helpful', 'satisfying', 'rewarding', and 'aesthetically pleasing'. Interfaces B and C were rated equally in terms of being 'enjoyable'. These ratings are consistent with the qualitative analysis presented above - that the real world metaphor makes Interface B easier to comprehend and work with, whereas the abstract parameters interface gives access to a wider range of novel interactive experiences and supports greater control and experimentation.

\subsection{Artists' Preferred Haptic Sensation}

A further question was whether there were particular haptic sensations that were clearly preferred by the artists in the study. Participants were asked to identify which pen tool and paper type combinations they preferred (Interface B), and which haptic cue combinations they preferred (Interface C). Each artist could state as many preferences as they wanted.

The preferences for pen tool and paper type combinations when evaluating Interface B are shown in Figure 9. 


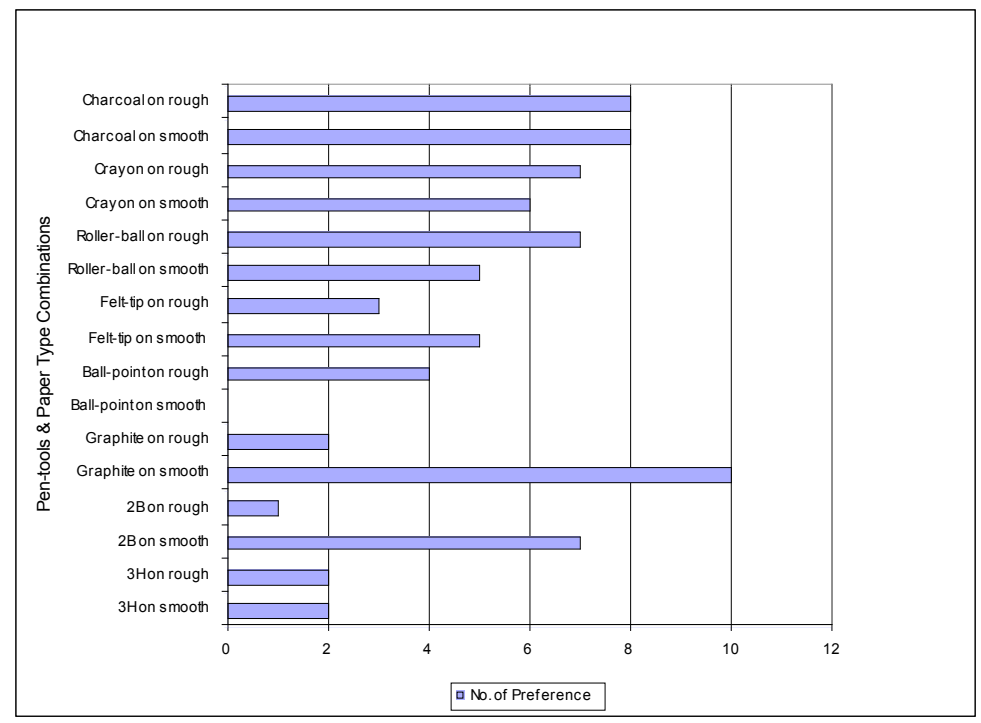

Figure 9: Pen-tool and Paper Type Preference

The scattered distribution of the artists' preferences towards the pen-tools and paper type combinations shows that none of these combinations dominated the artists' preference. One implication of this is that fixed haptic feedback (Interface A) would be unable to address the preferences of all users however well it was designed.

The artists tended to prefer the combinations for which they perceive the haptic feedback to be similar to a real world counterpart. In this case, two different patterns of reasons for haptic feedback preference were observed. The first was realism of tactile sensation as suggested by the metaphor used. For example, '2B pencil' on 'smooth paper' was perceived as similar to the actual drawing implements in the real world. The second was about realism of tactile sensation, but compared to a different tool. For example, 'charcoal' on 'smooth paper' was perceived by some as feeling like a pen on a whiteboard.

One contributing factor to 'ball-point pen' on 'smooth paper' not being preferred by any artists was that the haptic sensation did not meet the artists' expectations. For example, Artist 6 described this interaction as "a pen, which is stuck with ink". The interaction perceived still feels similar to the real world, but not in an ideal situation.

The preferences for haptic dimension cue combinations when evaluating Interface $\mathrm{C}$ are shown in Figure 10. Here, every combination is represented by the level of the dimension cues which are 'low', 'medium', and 'high' for scratchiness and stickiness, and 'low', and 'high' for bumpiness. For example, the combination 'high-high-low' indicates the haptic feedback of 'high' scratchiness, 'high' bumpiness, and 'low' stickiness. 


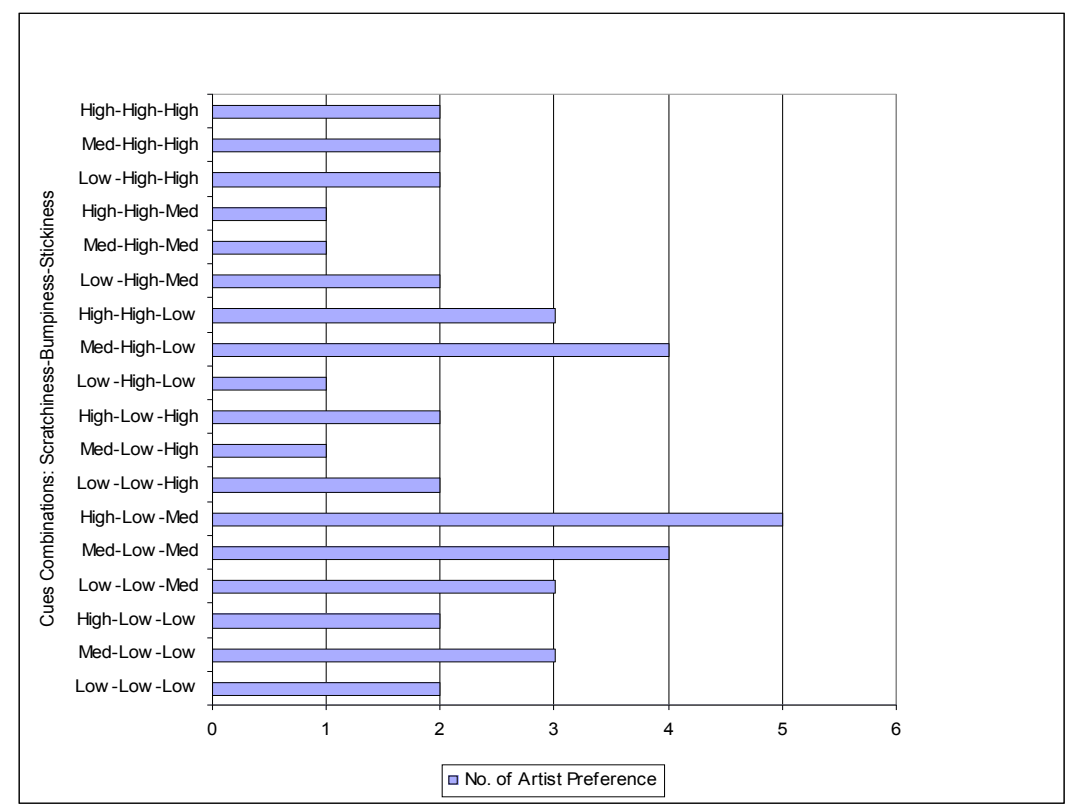

Figure 10: Haptic Dimension Cues Preference

The findings reveal a wide spread of preferences with respect to the haptic dimension cue combinations. These findings are consistent with those for Interface B. However, participants had more difficulty in identifying which combinations they preferred in Interface C than B. This is probably because of less familiarity with the tactile sensations in Interface $\mathrm{C}$ and less discriminability between the terms used. As an example, when asked on the haptic preference between Interfaces B and C, Artist 10 said: "Probably B because it's easier to remember how it felt whereas $C$ is different combinations which is quite confusing. It's difficult to tell what it is doing."

A pattern was identified when observing how an artist chose a particular haptic dimension cue combination. The analysis indicates that feedback perceived as similar to a real world haptic sensation tends to be preferred. In the case of Interface C, the artists tried to relate their new tactile experience with those that they had encountered in the real world. When trying the combinations of 'high-low-low', 'low-high-low', and 'medium-high-low', Artist 3 said: "I feel more pressure because it's more like using a chalk. So there is pleasure and it's scratching."

\section{Discussion}

The role of metaphor as a memory aid (Blackwell, 1998) has been applied in interface design. Reification of metaphor (Blackwell, 2006) can make the abstract haptic sensation from a drawing interaction concrete. During the evaluation, the artists utilised the visual metaphors presented on the interface to decide the tactile sensation that they preferred for a drawing interaction. The metaphor employed in the study influences the factors that affect user preference of tactile experience for a drawing application. Such factors are based on artists' familiarity with the real world interaction and user control, and a balance between a new experience and familiarity of the haptic sensation. The relevance of metaphor in presenting haptic information has led to a discussion of whether metaphor enhances or hinders user haptic experience in a drawing interaction. What suggestions would be appropriate to address the issues if metaphor hinders user experience?

\subsection{Metaphor Used Enhanced User Experience}

The evaluation study provides evidence that a metaphor enhances user haptic experience when the underlying haptic parameters match the sensation artists had expected. In the case of Interface A, it fails to convince artists of its "reality" in terms of the tactile sensation. Almost all artists in the evaluation study noticed this situation. In this case, the metaphor has helped the artists to differentiate the haptic feedback from Interface A with what they have experienced and understood in the real world. This gives a reason as to why either Interface B whose haptic metaphor linked to suggested pen tool or Interface $\mathrm{C}$ whose haptic metaphor is consistent with user control based on degree of bumpiness, scratchiness and stickiness is preferable to Interface A where there is no designed haptic metaphor.

The metaphor used for Interface B has helped the artists to build their own expectation of the tactile sensation to be felt based on their previous experience. This is in line with Wells and Fuerst's (2000) assertion that metaphor must be suitable and 
familiar to users to take advantage of previous domain knowledge and experience. The artists expected the underlying haptic parameters in Interface B to reflect reality. When such a prediction is met, artists tend to prefer the interface and also rated it as being helpful to them. Where the metaphor was not perceived in line with the haptic description, not all pen tools and paper type combinations were preferred by the artists.

The familiarity of the metaphor used for Interface B is a possible reason for this interface to have scored better in the 'helpful' criterion than Interface C. This is consistent with Wells and Fuerst (2000) and Vaananen and Schmidt (1994), who argue that concrete real-world-based object metaphors should be considered prominently because of their familiarity and attractiveness to most types of users, especially for first time and casual users of a system. This situation is parallel to the case of the artists who were first time users of the PHANToM and the haptic drawing system. This result also indicates that diagrams as used by Blackwell (1998), using pictures and text describing the images, are adequate as a visual metaphor to represent haptic information for a drawing application.

To date, little attention has been focused on user haptic experience in the design requirements of art haptic applications, as most work concentrates on the technical aspects of the interactions. This raises a concern that users may reject a product if it does not have the functionalities that they require. As presented in Section 2.2, users may not want to use a drawing system if it does not provide additional features to them. So is it necessary to mimic reality in terms of haptic sensation when developing a drawing application?

The study findings of the preference exercise revealed that in the case of Interface $\mathrm{C}$, the metaphor to represent the haptic sensation is acceptable to the artists. Artist 19 indicated his dislike of using a computer drawing application. He said: "I prefer to use the real pencils!" However, the fact that he preferred Interface $\mathrm{C}$ as his inferred final preference is an indication that the additional feature that is the intuitive haptic sensation may have persuaded him to like the interface. This correlates with Scali et al (2002) that artists only use computers if they provide additional functionalities that could support the tasks the artists are doing. This finding also implies that not only haptic feedback should be included in a design but a consideration of the way haptic information is presented to the users.

The metaphor for Interface $\mathrm{C}$ was designed by exploiting user experience in expressing haptic sensation when using drawing tools in the real world. The 'everydayness' concept helps in artists' learning and understanding the interface metaphor used in the prototype. Artist 9 noted the interface metaphor of Interface 3 is easy to learn. Her response correlates with Wells and Fuerst's (2000) that a metaphor must be suitable and familiar to the users to take advantage of users' previous domain knowledge and experience. Artist 9's remark is an example that indicates to some extent that the metaphor used in Interface $\mathrm{C}$, which does not involve a real world object-based metaphor, is also familiar to the users. Such familiarity, which corresponds to the way people describe a tactile sensation from an object they touch, seems to support such a familiarity feature in a haptic interface design.

Haptic exploration which occurs during a drawing interaction, enables the artists to feel the changes of the surface texture profiles implemented in the system. Their ability to feel these textures makes the drawing interaction more controllable and also creates a feeling of "making or doing something". The haptic sensation felt could be a reason both Interfaces B and C were rated equally in terms of being 'enjoyable' in Section 6.5. Such enjoyment for Interface B has been explicitly expressed by the artists from their subjective haptic experience presented in Section 6.3. Similarly, artists felt that the ability to directly manipulate and control the haptic parameters on Interface $\mathrm{C}$ is fun. Some artists thought that the visual metaphor used for the interface does not create any expectations for the tactile sensations to be felt, hence making the experimentation of the haptic feedback to generate different sensations more interesting. This situation may have led to the artists enjoying the interaction using Interface $\mathrm{C}$.

The fact that Interface $\mathrm{C}$ allows experimentation on the haptic sensation has resulted in some artists preferring the extreme sensations such as the 'high' scratchiness, 'high' bumpiness, and 'high' stickiness because the sensation has been associated to a feel of being freer and more creative. This could be a reason this interface was rated better than Interface B with respect to the "supportive of creativity" criterion. However, from the artists' subjective haptic experience, it could be argued that this criterion could also be applicable to Interface B because the haptic feedback provided has been described as helping artists to engage with their expressive feelings.

\subsection{Metaphor Used Hinders User Experience}

The study findings that explained the negative user haptic experiences show that at times the visual metaphor used to represent the haptic information could hinder user experience. One of the factors for such experience is when the visual metaphor used is perceived as less familiar to the artists. This situation comes across in a few cases in Interface $\mathrm{C}$ when artists compared the visual metaphor of the interface with those in Interfaces A and B. Despite some artists saying that Interface $\mathrm{C}$ could be easily learnt, some noted that it is more complex than Interface B. Interface $\mathrm{C}$ requires artists to 
construct their own media from the combination of haptic cues. Those who had reservations with Interface $\mathrm{C}$ did not particularly like this feature as they might not be able to relate the haptic sensation they know with a specific tool. When they found the haptic sensation that they preferred to use, it was still difficult to remember the combination as compared to the pen tool and paper type metaphor used in Interface B.

The complexity of the metaphor used in Interface C, as perceived by some artists, suggests proceeding with caution in designing future haptic interfaces. Based on the artists' responses when interacting with the interface, suggestions to be considered include allowing users to create memorable tools from the haptic cues combinations, for ease of memory and to link back to the real world, creating a single integrated interface employing both metaphors. For example, Artist 5 preferred Interface $\mathrm{B}$ but offered an adaptation for using Interface $\mathrm{C}$ to facilitate remembering preferred combinations of haptic cues. She said: "If there is an easy way to remember or 'low-low-high' is a nice one, make it as a tool, create a tool, I mean Interface C." Besides improving the interface, allowing more time to the user interacting with the system would probably change their attitudes towards Interface C. It should be highlighted that all artists in the evaluation study were using the PHANTOM for the first time and were only given 15 minutes to interact with Interface C. These study findings indicate that with careful design, the metaphor used in the interface could provide positive user experience during a drawing interaction.

The metaphor used for Interfaces A and B has built up an expectation of the haptic sensation to be perceived as well as the visual lines when using the pen tools. The expectations on the visual cues are not the focus of the research.

When experiencing the haptic feedback suggested by the visual metaphor, the haptic sensation perceived in Interface A has resulted in a perception that it is easier to make a mark on the interface as compared to Interface B, despite the fact that the visual effect for both Interfaces A and B has been implemented in the same manner. In the case of Interface A, the visual effect becomes apparent whereas it does not in Interface B. This relates to Lederman and Hamilton (2002) on the role of haptics when a visual cue is present. When interacting with Interface A, that feels smooth throughout the interaction as perceived by most artists, visual perception dominates; hence, the perception of macrogeometry (i.e. the shape of the line drawn) is more obvious.

When experiencing the haptic feedback for Interface B, the variation of sensation with respect to the suggested metaphor has made the perception of microgeometry (i.e. texture) become more dominant than the perception of macrogeometry. As a result, the artist tends to focus on a specific haptic feature and expect a differentiation of line effects when using different pen-tools. To some extent the visual effects received may have also influenced artists' judgement in assessing the haptic feedback of the object-based metaphor interfaces. They tend to associate the mark produced with the pressure that they applied on the drawing surface and the haptic feedback expected from the interaction. The fact that the prototype does not correspond to realistic interaction behaviour disappoints some artists when using Interface B that is designed to mimic real world sensation.

\subsection{Addressing the limitations}

In Section 2.1, the development of creative artwork such as $\mathrm{dAb}$ involves a haptic rendering technique that takes into consideration the pressure and speed of a painting interaction. Replicating this technique to simulate haptic feedback in the prototype may result in addressing the haptic sensation with respect to the pressure applied during an interaction. In theory, having better haptic rendering will better replicate reality. This could probably address responses from artists who felt that the tactile sensation only partially mimics reality. This could involve a better implementation of the haptic prototype in which the differentiation of the haptic feedback received between the 'press' and 'push' actions as indicated in the preliminary taxonomy of haptic features for the drawing domain could be addressed. However, how much reality is needed to satisfy user requirements?

Artist 20 provides an insight into answering this issue of satisfying a haptic user requirement. When evaluating 'charcoal' on 'smooth paper' in Interface 2 he commented: "I guess no matter how you sort of try to compensate this in programming the machine you know there is still no way that you actually make a charcoal pencil" In relation to this Bordegoni et al (2001) noted our ability to distinguish point-like events from force feedback generated by a PHANToM and to mentally integrate in time the continuity of a sequential signal, enabling us to feel the haptic sensation when touching the surface. If we are able to exploit human weaknesses in perceiving haptic feedback, we may still be able to present haptic information that meets users' requirements. Hayward et al (2004) noted that users are able to adapt to the imperfection of a rendering technique to recognise a particular haptic sensation. Hayward's argument has been seen in some of the artists' responses. In this case, users experience could be a way to decide to what extent reality is needed to mimic the haptic sensation. 


\subsection{Some Reflections on Generalising from the Study Findings}

The haptic drawing prototype evaluated in the study was represented using three interfaces. Interfaces A and B shared the same visual abstraction of pen tool and paper type features displayed on the screen, while Interface $\mathrm{C}$ has the textual description of haptic feedback with a specific level of sensation, namely 'low', 'medium', and 'high'. Improvements made on these interfaces could, for example, include real images of pen tools and paper type surfaces as used in the study described in Section 3.1 for Interfaces A and B, a slider version to represent the degree of haptic sensation for Interfaces C, and a better haptic rendering as described in the earlier section for all three interfaces. Such improvements to the appearance of the interfaces may affect user experience whilst interacting with the interfaces. This prediction is revealed from one particular artist described in Section 6.4 who enjoyed the tactile sensation of pen-tools on rough paper in Interface B. The visual appearance of the rough paper on the computer screen may have influenced this artist's perception of haptic feedback. This situation indicates that a particular set of user preferences could be obtained based on a specific set of interfaces. In this research project, minimum feasible changes between the interfaces were made in order to focus on the matter of concern i.e. the haptic metaphor. This is seen in the dependency of the development of haptic sensations in Interface $\mathrm{C}$ that was based on Interface B. The focus on haptic metaphor was intended to obtain study findings that reflect the artists' responses towards the haptic features rather than other elements of the different interfaces. However, since many aspects of context can influence subjective data, it is impossible to be certain how the study findings generalise. By minimising the non haptic changes between the interfaces, it is aimed to maximise the chances of the findings generalizing.

\section{Conclusion}

Past work on haptic interactions has generally focused on implementation details and accurate haptic rendering. Our work has taken a different focus, namely on the user's interaction experience, on whether user controllable haptic feedback is valued by artists in a drawing tool, and on how to support user control at the interface.

The two interface metaphors implemented were liked approximately equally, but for different reasons. The haptic interface implementing a real world metaphor was valued for its familiarity, and was considered helpful. In contrast, the abstract metaphor interface was considered to better support creativity. However, some participants found it harder to understand. They also found it difficult to remember which combinations of stickiness, bumpiness and scratchiness they preferred. Within the two interfaces where the user could control the haptic sensation, there was no single preferred sensation, though ones for which there was a real-world analogue (not necessarily explicit in the metaphor) were generally preferred to those with less familiar sensations. These findings show that an interface that combines both metaphors, allowing users to work with analogues of real-world tools, to work directly with the abstract haptic dimensions or to define their own tools in terms of those dimensions, would be a promising design solution.

The fact that haptic feedback was the focus of this research project has made the visual features secondary to the work. To address this matter, future studies should include the role of the visual feedback (and particularly the way visual and haptic feedback combine to create particular user experiences); the role of sound, orientation and other contextual factors that contribute to the overall user experience; and the details of the designs of the user control (e.g. sliders would give greater control than buttons).

Overall, current haptic technologies are still relatively primitive (the PHANTOM has single point contact, is much more unwieldy than the typical pen or crayon, generates motor noise, etc.). Nevertheless, this study has provided evidence that haptic feedback can enhance artists' creative experience with computer-based drawing applications. Two different interface metaphors were found to give similarly positive experiences, and an interface that combined both would be a very promising design offering.

\section{References}

1. Baxter B., Scheib V., Lin M.C., Manocha D. (2001), DAB: Interactive Haptic Painting with 3D Virtual Brushes. In Proc. ACM SIGGRAPH 2001. 461-468.

2. Baxter W., Wendt J., Lin M.C. (2004), IMPaSTo: A Realistic, Interactive Model for Paint. In Proc. of NPAR 2004, The $3^{\text {rd }}$ International Symposium on Non-Photorealistic Animation and Rendering. 45-56.

3. Blackwell A.F. (1998), Metaphor in Diagrams, Unpublished PhD Thesis. Cambridge University, UK.

4. Blackwell, A.F. (2006), The Reification of Metaphor as a Design Tool, ACM Transactions on CHI. 13. $490-530$.

5. Bordegoni, M., Cugini, U. and Mussio, P. (2001), Issues in the Combination of Visual and Haptic Interaction, $1^{\text {st }}$ Int. Conf. on Universal Access in HCI. 
6. Brewster S.A. (2005), The impact of haptic 'touching' technology on cultural applications. In Hemsley, J., Cappellini, V. and Stanke, G. (eds.) Digital Applications for Cultural Heritage Institutions. Ashgate Press, UK. 273-284.

7. Burdea, G.C. (2000), Haptic Issues in virtual Environment, In Proceedings of Computer Graphics International 2000, Geneva, Switzerland, June 2000, IEEE 2000

8. Castle, G., Adcock, M., and Barrass, S. (2002), Integrated Modal and Granular Synthesis of Haptic Tapping and Scratching Sounds, EuroHaptics 2002.

9. Foskey, M., Otaduy, M., Lin, M.C. (2002), ArtNova: Touch-enabled 3D Model Design, Proceedings of the IEEE Virtual Reality Conference. 119-126.

10.GHOST SDK (General Haptics Open Software Toolkit), (1999), Programmer's Guide, Version 3, SensAble Technologies, Inc., Chapter 3.

11. Guerraz A., Loscos C., Ritter Widenfeld H. (2003), How to Use Physical Parameters Coming From the Haptic Device Itself to Enhance the Evaluation of Haptic Benefits in User Interface. In Proc. Eurohaptics 2003, Trinity College Dublin and Media Lab Europe, July 6th - 9th 2003.

12. Guichard Meili, J. (1984), "Matisse Paper Cutouts", Thames and Hudson Ltd., London.

13.Hayward, V., Astley, O.R., Cruz-Hernandez, M., Grant, D., and Robles-De-La-Torre, G. (2004), Tutorial: Haptic Interfaces and devices, Sensor Review, Volume 24, No. 1, Emerald Group Publishing limited.

14.Iinuma, T., Murota, H., Kubota, Y., Tachihara, H. (1999), Representation of the Tactile Surface Texture of an Object Using a Force Feedback System, ACM SIGGRAPH 1999, 274.

15.Kim, L., Sukhatme, G.S., Desbrun, M. (2003), Haptic Editing of Decoration and Material Properties, In $11^{\text {th }}$ Symposium on Haptic Interfaces for Virtual Environment and Teleoperator Systems (HAPTICS'03), 213-220.

16.Klatzky,R.L., Lederman,SJ (2002), Perceiving Texture Through a Probe, in McLaughlin, Hespanha, \& Sukathme (eds), Touch in virtual environments, Prentice Hall PTR, 180-193.

17.Lederman, S.J. and Hamilton, C. (2002), Using Tactile Features to Help Functionally Blind Individuals Denominate Banknotes, Human Factors, Vol. 44(3), pp. 413 - 428.

18.Lin M.C., Baxter B., Foskey M., Otaduy M.A., Scheib V. (2002), Haptic Interaction for Creative Processes with Simulated Media. In Proc. IEEE Int. Conf. Robotics \& Automation. 598-604.

19. MacLean K.E. (2000), Designing with Haptic Feedback. In Proc. of IEEE Robotics and Automation (ICRA'2000), San Francisco, CA, Apr 22-28.

20. Masden K.H. (2000) Magic by Metaphors. DARE 2000, April, 2000, Elsinore, Denmark, ACM. 167-169.

21.McGee, M.R., Gray, P.D., Brewster, S.A. (2001), Feeling Rough: Multimodal Perception of Virtual Roughness, In Proceedings of Eurohaptics 2001 (Birmingham, UK), 29-33.

22. Nakakoji, K. and Yamamoto, Y. (2003), Towards A Taxonomy of Interaction Design Technoques for Externalizing in Creative Work, $10^{\text {th }}$ International Conference on Human-Computer Interaction (HCII2003), Crete, Greece, June, 2003

23. Preece J., Rogers Y., Sharp H. (2002), Interaction Design, John Wiley \& Sons.

24. Scali, S., Shillito, A.M., Wright, M. (2002), Thinking In Space: Concept Physical Models and The Call For New Digital Tools, Crafts in the $20^{\text {th }}$ Century, Edinburgh.

25. Shillito A.M., Paynter K., Wall S., Wright M. (2001), 'Tacitus' Project; Identifying Multi-Sensory Perceptions in Creative 3D Practice for Development of a Haptic Computing System for Applied Artists. In Proc. of Eurohaptics 2001.

26. Snibbe S., Anderson S., Verplank B. (1998), Springs and Constraints for Haptic Sculpting. In Proc. of the Third PHANTOM Users Group Workshop, AI Lab Technical Report No.1643, MIT.

27. \{removed for blind review purpose\}, Haptic Cues for Supporting Interaction Design in the Drawing Domain. In Proc. of BCS HCI 2004, Vol 2, (Leeds, UK).

28. Vaananen K., Schmidt J. (1994), User Interfaces for Hypermedia: How to Find Good Metaphors? Conference Companion, CHI '94, Boston, Massachusetts, USA. 263-264.

29. Wall, S.A., Harwin, W.S. (2000), Interaction of Visual and Haptic Information in Simulated Environments: Texture Perception, In Proceedings of Workshop on Haptic Human Computer Interaction, $31^{\text {st }}$ August $-1^{\text {st }}$ September, Glasgow, Scotland, $39-44$

30.Wells, J.D., Fuerst, W.L. (2000), Domain-Oriented Interface Metaphors: Designing Web Interfaces for Effective Customer Interaction, Proceedings of the $33^{\text {rd }}$ Hawaii International Conference on System Sciences, IEEE. 6047. 
31.Yeh, J., Lien, T., Ouhyoung, M. (2002), On the Effects of Haptic Display in Brush and Ink Simulation for Chinese Painting and Calligraphy, Proceeding Pacific Graphics 2002 (PG 2002), Beijing, China. 439.

32. Yu W., Brewster, S.A. (2003), Evaluation of multimodal graphs for blind people. Journal of Universal Access in the Information Society. 105-124.

33.Yu, W., Ramloll, R., Brewster, S.A. (2001), Haptic Graphs for Blind Computer Users, Brewster, S.A. \& Murray-Smith, R. (eds.), Haptic Human-Computer Interaction, Springer LNCS, Vol. 2058, 41-51. 